\title{
Statistical Study of Globules Projected on Stellar Clouds
}

\author{
D. A. Rojkovsky \\ Astrophysical Institute, Alma-Ata, U.S.S.R.
}

$\mathrm{T}$ HE discovery of globules by Bok and Reilly leads to various speculation concerning their cosmogonical meaning as protostellar bodies in our galaxy.

It is well known that the most favorable conditions for the discovery and study of globules are reached when they are connected with diffuse galactic nebulae (Dufay $\left.{ }^{2(a)}\right)$. Observations show that in this case globules possess various and generally irregular shapes. It is quite probable that these globules are connected with large dark clouds, genetically linked with bright nebulae..$^{2-5}$ The number of globules in a nebula depends essentially on the peculiarity of its structure. Nevertheless the total area of the sky covered by the nebulae is relatively small and their physical conditions depending on nearby hot stars are peculiar. Consequently it is hardly possible to suppose that the properties of globules present in nebulae are the same as in the other regions of the galaxy. It is important to collect more data concerning the spatial distribution of globules, their density, sizes etc. The stellar clouds of the Milky Way give some possibility for further study in this respect. In the case of a sufficiently dense stellar field the projecting globules can be discovered as some fluctuations in the stellar population as observed on the photograph.

My method of the determination of optical density, number and angular dimension of globules, contained in a given area, is based on the application of the Laplace distribution, justifiable for the accidental arrangement of stars in the field, namely

$$
\nu_{N}^{(n)}=\frac{N !}{n !(N-n) !} p^{n-1}(1-p)^{N-n}
$$

where $N$ is the total number of stars up to $m$ th magnitude in a given area $S$ of the stellar field, $n$ is the number of stars in the area $\sigma, p=\sigma / S \ll 1$, and $\nu_{N}{ }^{(n)}$ is the number of areas $\sigma$ containing $n$ stars.

The applicability of (1) was verified by counts in artificial, as well as in natural fields of stars. In the latter case, areas located in high galactic latitudes were

\footnotetext{
1 B. J. Bok and E. F. Reilly, Astrophys. J. 105, 255 (1947).

2 (a) J. Dufay, L'Astronomie (October, 1954); (b) Nebuleuses galactiques et matière interstellaire, 396, Paris.

3 V. G. Fessenkov and D. A. Rojkovsky, Astron. Zhur. 29, 381 (1952).

${ }^{4}$ D. A. Rojkovsky, Compt. rend. acad. sci. U.R.S.S. 98, 553 (1954).

6 B. J. Bok, Astron. J. 61, 146 (1956).
}

considered, in which the influence of absorbing matter is quite insignificant.

Figure 1 illustrates the precision with which the Laplace distribution (dots) satisfies the observed distribution of stars (crosses).

In the case of a random distribution of $M$ equal globules over a considerable area, the number of stars in different areas is given by the expression

$$
\nu_{N_{1}}{ }^{(n)}=\nu_{N}{ }^{(n)}+M p\left\{\nu_{N}{ }^{[n /(1-\gamma)]}-\nu_{N}{ }^{(n)}\right\}
$$

with

$$
\gamma=\int_{\left(m_{1}-m_{0}\right)}^{m_{1}} a(m) d m
$$

where $m_{0}$ is the mean absorbing capacity of globules expressed in stellar magnitudes, $m_{1}$ is the limiting magnitude of stars still discernible on the photograph, and $a(m)$ is the distribution function of stars according to their magnitudes.

With sufficiently large values of $m_{0}$ and $M$ the graph of the function (2) must have two maxima:

$$
\begin{aligned}
& \text { principal maximum: } \nu_{N}{ }^{(\langle n\rangle)}[1-M p \gamma], \\
& \text { secondary maximum: } M p \nu_{N}{ }^{(\langle n\rangle)}
\end{aligned}
$$

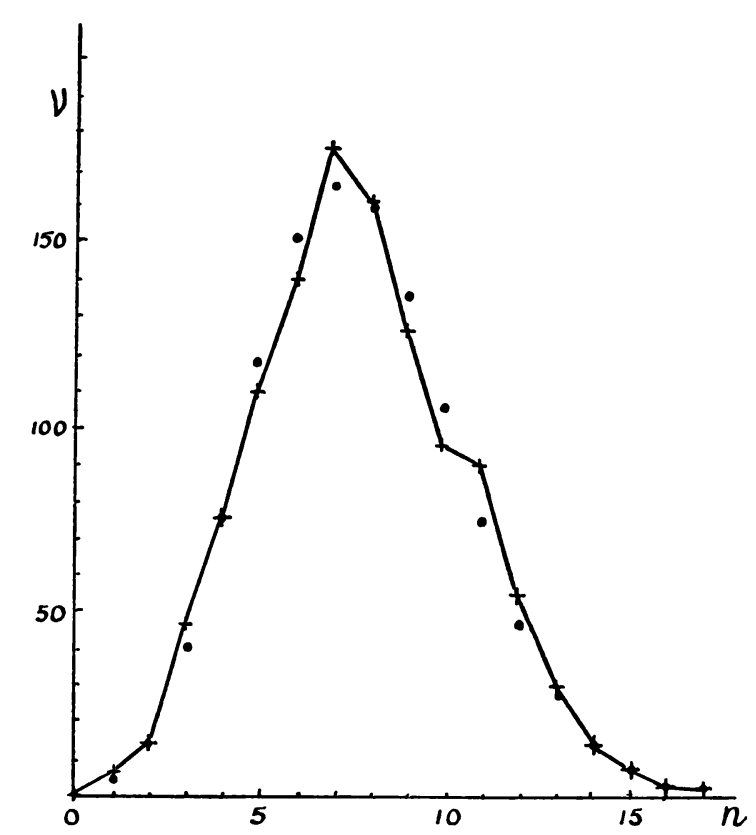

FIG. 1. Comparison of Laplace distribution [dots] with observed distribution of stars [crosses] for regions having no absorbing material. 


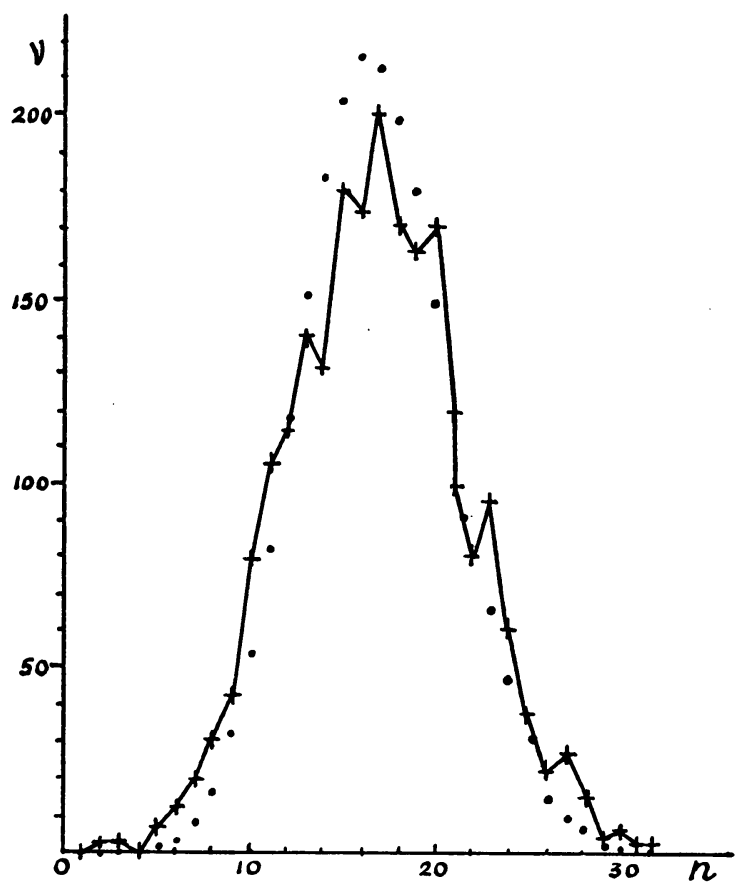

FIG. 2. Comparison of Laplace distribution [dots] with observed distribution of stars [crosses] for the region $l=2^{\circ}, b=-1^{\circ}$ where some absorbing globules exist.

with corresponding modes $\langle n\rangle$ and $\langle n\rangle(1-\gamma)$. The actual star counts can be presented graphically. Considering the shape of the curve one can determine the values $M$ and $m_{0}$ as mean characteristics of the globules under consideration. The homogeneous star fields in the neighborhood of the dark clouds are most suitable for this investigation. Star counts were carried out by the author for three analogous areas. It is found that in general the observed distribution of the stars agrees well with (1). The deviations are within the limits of admissible errors. The distribution curves have no secondary maxima.

A typical case presents apparently an area with coordinates $l=2^{\circ}, b=-1^{\circ}$ chosen in the direction of the galactic center. The diagram (Fig. 2) represents the comparison between the observed distribution (crosses) and the calculated distribution (dots) according to formula (1).

As the values $m_{0}$ and $M$ are not very large here, their evaluation can be made only approximately. The observed discrepancy can be accounted for, if in this case globules of dimensions nearly $1^{\prime}$ occupy only 10 to $12 \%$ of the whole area, their number being equal to $10^{2}$, with $\gamma<0.5\left(m_{0}<1\right)$.

The application of selected star counts permits one to deduce the following conclusions:

(1) In cases when the apparent density of the star field is not very great and the method given before is applicable, small statistical deviations can be accounted for by the presence of globules. Their number must be of the order of $10^{2}$ per square degree, angular dimensions about $1^{\prime}-2^{\prime}$ and mean absorbing capacity smaller than $1 \mathrm{mg}$. The number of quite opaque globules must be apparently less by 2 orders.

(2) Stellar clouds of high density and vast dimensions are more appropriate to researches on very small globules, namely, about $1^{\prime}$ or less in diameter. In this case only isolated and big globules are observed.

The results obtained by the author in 1955-1956 somewhat contradict a recently published communication ${ }^{7}$ concerning the same problem. The cause of the discrepancy is not clear and merits further investigation.

${ }^{6}$ D. A. Rojkovsky, Bull. Astrophys. Inst. Acad. KazSSR (to be published).

${ }^{7}$ R. Fleischer and P. Conti, Astron. J. 61, 4 (1956). 